\title{
Electrical load forecasting through long short term memory
}

\author{
Debani Prasad Mishra ${ }^{1}$, Sanhita Mishra ${ }^{2}$, Rakesh Kumar Yadav ${ }^{1}$, Rishabh Vishnoi ${ }^{1}$, \\ Surender Reddy Salkuti ${ }^{3}$ \\ ${ }^{1}$ Department of Electrical Engineering, IIIT Bhubaneswar, Bhubaneswar, India \\ ${ }^{2}$ Department of Electrical Engineering, KIIT Deemed to be University, Odisha, India \\ ${ }^{3}$ Department of Railroad and Electrical Engineering, Woosong University, Daejeon, Republic of Korea
}

\begin{abstract}
Article Info
Article history:

Received May 20, 2021

Revised Nov 6, 2021

Accepted Nov 27, 2021

\section{Keywords: \\ Daily load curve \\ Factors affecting load \\ Long short term memory \\ Monthly load curve \\ Recurrent neural network \\ Root mean square deviation}

ABSTRACT

For a power supplier, meeting demand-supply equilibrium is of utmost importance. Electrical energy must be generated according to demand, as a large amount of electrical energy cannot be stored. For the proper functioning of a power supply system, an adequate model for predicting load is a necessity. In the present world, in almost every industry, whether it be healthcare, agriculture, and consulting, growing digitization and automation is a prominent feature. As a result, large sets of data related to these industries are being generated, which when subjected to rigorous analysis, yield out-of-the-box methods to optimize the business and services offered. This paper aims to ascertain the viability of long short term memory (LSTM) neural networks, a recurrent neural network capable of handling both longterm and short-term dependencies of data sets, for predicting load that is to be met by a Dispatch Center located in a major city. The result shows appreciable accuracy in forecasting future demand.
\end{abstract}

This is an open access article under the CC BY-SA license.

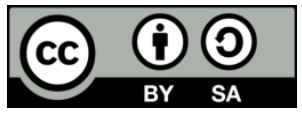

\section{Corresponding Author:}

Surender Reddy Salkuti

Department of Railroad and Electrical Engineering, Woosong University

Jayang-Dong, Dong-Gu, Daejeon, Republic of Korea

Email: surender@wsu.ac.kr

\section{INTRODUCTION}

Electricity is an extremely important source of energy and plays a significant role in a country's economic development [1]. Load forecasting is necessary for the proper functioning of electrical dispatch centers. Load forecasting is a method used to maintain synchronicity of demand and supply of electrical power. With a greater contention for the market and greater decentralization, short-term forecasting is becoming more significant [2]. In an age where smart grids with advanced sensing and communication are fast becoming a reality, load forecasting is a field where the scope and necessity of accuracy are increasing day by day [3]. Numerous significant decisions depend upon the load forecasts like economic dispatch, distribution schedule, schedule of protection, and maintenance measures [4]. From proper maintenance of equipment to the economic strategies of the suppliers, load forecasting has a significant impact [5]. Especially for small-scale consumption units, peak load forecasting is very important [6]. Moreover, there has been an increased tendency of winters being colder and summers being more extreme than before. Therefore, greater use of equipment like air conditioners and heaters, and their use has become even more frequent [7]. This has led to more swings in terms of peak load and minimum load.

Many factors impact electrical load, their interrelation is complex and so is the extent to which one factor overrides one another. The factors can be divided into three categories [8]. Climate is considered the most important factor [9]. The short-term factors: They are factors that last only a little duration, like a sudden weather change. The middle-term factors: They last for a substantial duration and have a distinct 
characteristic that governs the corresponding load variation. For example, seasonal climatic variations. The long-term factors: They last for a significant time period, and usually over multiple forecasting periods [10]. For a particular area, the temperature is the measure of the average warmth or coolness of the surrounding. Temperature is far more influential than other factors like wind speed and cloud cover [11].

When the temperature falls to a certain extent, it becomes cold and households require more energy [12]. Similarly, after a temperature rise, more energy is required. Both in summers and winters, there is a strong correlating contribution between temperature and load curve. There is positive co-relation for summers, i.e. with temperature rise in summer leading to increased consumption of electrical load as appliances such as fans, coolers, and air conditioners (ACs), are turned on and if in summer the temperature falls, the same appliances are turned off for lesser load consumption. But there is a negative co-relation for winters, as only when the temperature falls, appliances used to keep the households warm are used. Generally, it can be seen, on working days there are substantial differences in load demands compared to working days and Weekends. There's lower consumption on Tuesdays to Thursdays while on weekends and days closer to weekends such as Mondays and Fridays the consumption is higher [13]. Another trend that can be observed is that on moving holidays: Holidays which do not have any fixed date, e.g. the religious festivals, also impact the forecast. Generally, on days of festivals, the demand is relatively higher. But since industrial activities are lesser, the overall consumption prediction becomes difficult.

In a broad sense, there are two types of models proposed or used for predicting future electrical demands, conventional statistical techniques, and artificial intelligence (AI) based techniques. Classic models use historical data and process them, and the estimates of parameters in such models can be easily interpreted. The models and techniques that fall under this category include auto regressive moving average (ARIMA) model [14], the regression seasonal ARIMA generalized autoregressive conditional heteroskedastic (Reg-SARIMA-GARCH) model [15], support vector machine models [16]. Time series model for series exhibiting multiple complex seasonalities (TBATS) [17]. AI techniques on the other hand prove to be more flexible due to their ability to adapt to moving data. The AI functions are nonlinear and nonparametric. In general, the AI models yield better results than traditional ones [18]. Neural networks and deep learning models have proven to be more accurate for electrical load forecasts than the traditional model. With the advent of smart grids and the ever-diversifying application of data analytics, a huge amount of data inflow and ever-increasing applications based on their analysis are expected [19], [20]. AI and machine learning techniques are expected to find a variety of uses not only in load forecasting but also in theft detection [21], protection and safety of nuclear [22] and thermal power plants [23] along with power price determination [24].

This paper has attempted to explore the implementation of relatively newer AI techniques in the domain of electrical load forecasting. Forecasting for electrical loads is a complex process that is prone to slight errors even when utmost care is taken in choosing the methods. This occurs due to the multiple factors influencing load patterns. Even such slight errors can lead to grave consequences to power system equipment and also gravely impact economic interests. These patterns are sometimes completely independent of each other and thus it becomes inherently impossible to find a co-relation. This paper inspects the use of the long short term memory (LSTM) model to solve this complex problem. For the same, we have to ensure that the data set on which this study is to be done, is organically dynamic and encapsulates the impact of all the factors. To achieve this, we use data taken from a major Dispatch Center, State Load Despatch Center (SLDC) State Load Dispatch Center, located in Delhi, one of the biggest cities of one of the biggest cities in the world in terms of active consumers. This paper, therefore, inspects the applicability of the LSTM model in load forecasting over a dynamic consumer base. This can create a platform for further exploration of the problem through LSTM using optimizers and supporting mechanisms. LSTM proves to be appreciably viable in handling the complex problem that electrical load forecasting presents.

\section{RESEARCH METHOD}

Our focus in this paper was to use the LSTM model to correctly predict the electrical load. LSTM is often used for time series forecasting, we chose to test how accurate it is for electrical load forecasting. To implement the algorithm on organic and potent data set, we scrapped the site state load dispatch centre, Delhi. We scrapped through the data from the $28^{\text {th }}$ of January to the $28^{\text {th }}$ of February. Parameters for each epoch of model development and training are presented in Table 1. From Table 1, 20 epochs were taken, with a batch consisting of 4600 data points. For cross-validating, 10 epochs were taken. The epochs and batch size were decided based on calculations and then approximated by trial and error for the best possible result. After the forecasts, root mean square error is used to compare the actual load to the forecast done by the model and received satisfactory results. 
Table 1. Parameters for each epoch of model development and training

\begin{tabular}{|c|c|c|c|c|c|c|c|}
\hline EPOCHS & $\begin{array}{l}\text { Time taken and } \\
\text { Time per step }\end{array}$ & Loss & $\begin{array}{l}\text { Value } \\
\text { Loss }\end{array}$ & EPOCHS & $\begin{array}{l}\text { Time taken and } \\
\text { Time per step }\end{array}$ & Loss & $\begin{array}{l}\text { Value } \\
\text { Loss }\end{array}$ \\
\hline 1 & $8 \mathrm{~s} \quad 1 \mathrm{~ms} / \mathrm{step}$ & 0.0320 & 0.0190 & 11 & $6 \mathrm{~s} 1 \mathrm{~ms} / \mathrm{step}$ & 0.0088 & 0.0074 \\
\hline 2 & $6 \mathrm{~s} 1 \mathrm{~ms} / \mathrm{step}$ & 0.0235 & 0.0137 & 12 & $6 \mathrm{~s} 1 \mathrm{~ms} / \mathrm{step}$ & 0.0087 & 0.0073 \\
\hline 3 & $6 \mathrm{~s} 1 \mathrm{~ms} / \mathrm{step}$ & 0.0160 & 0.0094 & 13 & $6 \mathrm{~s} 1 \mathrm{~ms} / \mathrm{step}$ & 0.0086 & 0.0072 \\
\hline 4 & $6 \mathrm{~s} 1 \mathrm{~ms} / \mathrm{step}$ & 0.0112 & 0.0091 & 14 & $6 \mathrm{~s} 1 \mathrm{~ms} / \mathrm{step}$ & 0.0085 & 0.0071 \\
\hline 5 & $6 \mathrm{~s} 1 \mathrm{~ms} / \mathrm{step}$ & 0.0103 & 0.0088 & 15 & $7 \mathrm{~s} 1 \mathrm{~ms} / \mathrm{step}$ & 0.0084 & 0.0070 \\
\hline 6 & $6 \mathrm{~s} 1 \mathrm{~ms} / \mathrm{step}$ & 0.0099 & 0.0085 & 16 & $9 \mathrm{~s} 1 \mathrm{~ms} / \mathrm{step}$ & 0.0084 & 0.0069 \\
\hline 7 & $6 \mathrm{~s} 1 \mathrm{~ms} / \mathrm{step}$ & 0.0096 & 0.0082 & 17 & $7 \mathrm{~s} 1 \mathrm{~ms} / \mathrm{step}$ & 0.0083 & 0.0069 \\
\hline 8 & $6 \mathrm{~s} 1 \mathrm{~ms} / \mathrm{step}$ & 0.0093 & 0.0080 & 18 & $7 \mathrm{~s} 1 \mathrm{~ms} / \mathrm{step}$ & 0.0083 & 0.0068 \\
\hline 9 & $6 \mathrm{~s} 1 \mathrm{~ms} / \mathrm{step}$ & 0.0091 & 0.0078 & 19 & $6 \mathrm{~s} 1 \mathrm{~ms} / \mathrm{step}$ & 0.0082 & 0.0067 \\
\hline 10 & $6 \mathrm{~s} 1 \mathrm{~ms} / \mathrm{step}$ & 0.0090 & 0.0076 & 20 & $7 \mathrm{~s} 1 \mathrm{~ms} / \mathrm{step}$ & 0.0082 & 0.0067 \\
\hline
\end{tabular}

\subsection{The LSTM model}

Traditional neural networks cannot use the concept of memory. They can't use the knowledge of previous states. This is a major drawback. Recurrent neural networks (RNN) in terms of architecture is not that different from the conventional neural network. An RNN however is capable of learning from memory. Figure 1 shows traditional neural networks, it is clear that since the output of neural networks doesn't loop back to previous layers, the previous states have no contribution towards future ones. Figure 2 shows a simple RNN, with a loop. Recurrent neural networks have proved to be powerful and accurate in their application. LSTM is a slightly different kind of RNN that overcomes some shortcomings of the standard version.

LSTM does not suffer from the short-term dependency problem of usual RNNs. Recurrent neural networks tend to prove inefficient when data shows more long term dependency than short term. LSTM does not have this issue and is considered suitable for time series modelling. LSTMs like RNN have a chain-like structure. However, in LSTM the repeating module has a slightly more complex structure. Each module has 4 layers and each layer interacts with one another. From Figure 3, it can see a cell state, represented by the top line running through the entire chain. The cell state only involves a few linear interactions. LSTM repeating module can either attach or delete the information running in the cell state. This is achieved through a "Gate". Gate is maintained or changes the cell state.

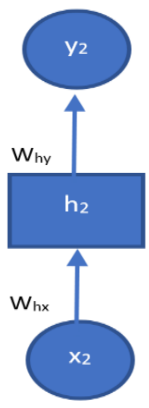

Figure 1. Traditional neural network

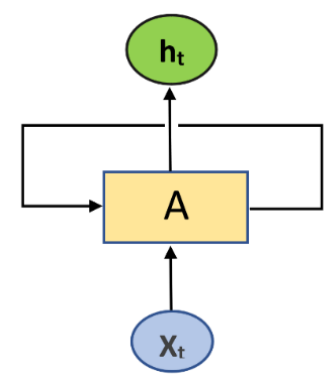

Figure 2. RNN diagram

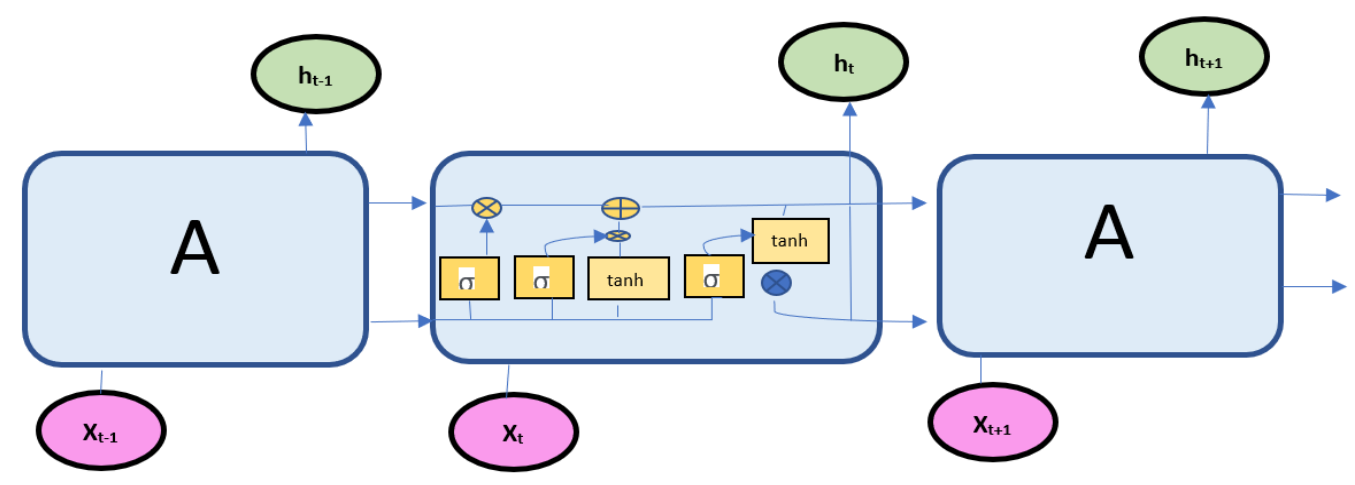

Figure 3. Basic structure of LSTM

Indonesian J Elec Eng \& Comp Sci, Vol. 25, No. 1, January 2022: 42-50 
Figure 4 shows how one of the reasons LSTM is different from RNN is the absence of a cell state. The cell state is the key to LSTM's ability to recognize short-term patterns. Figure 5 highlights the initial step, which is to determine if the incoming information from the cell state is to be deleted or not.

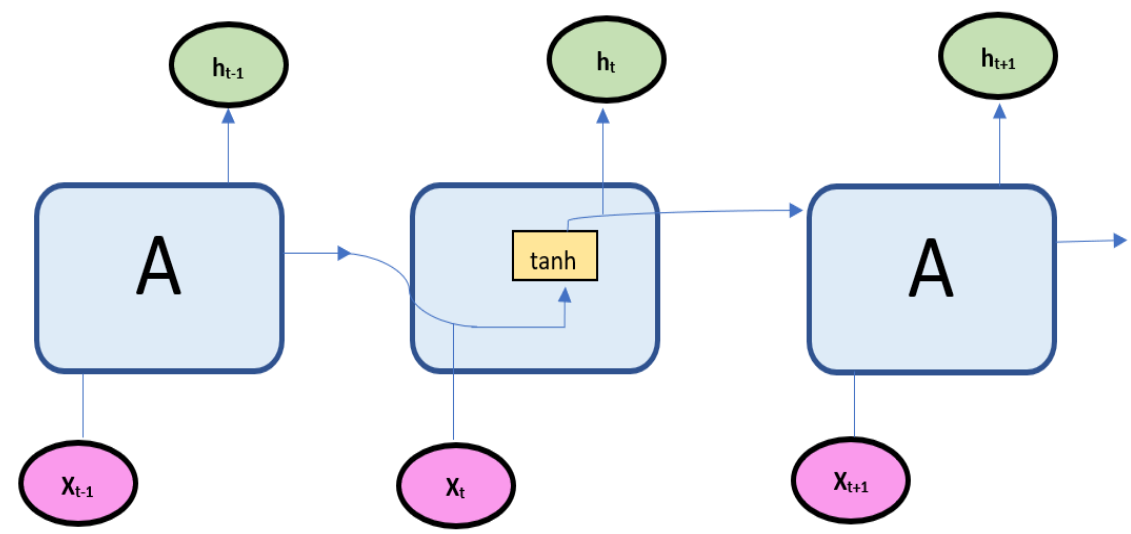

Figure 4. RNN does not have the cell states

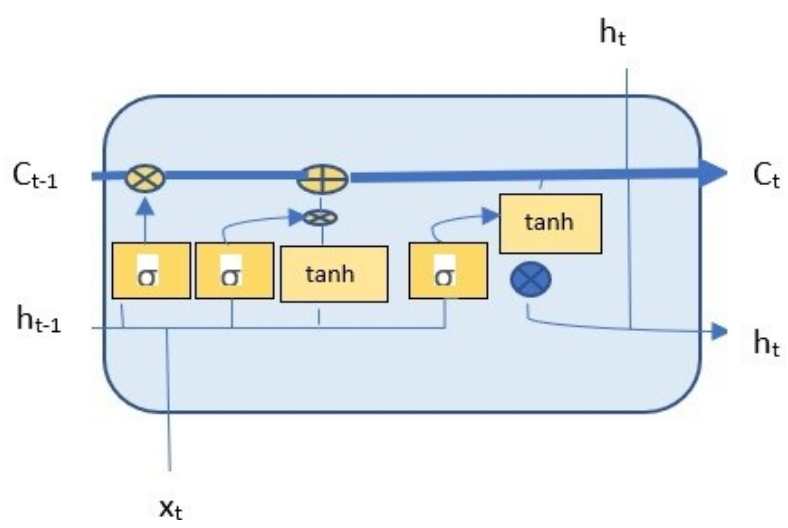

Figure 5. Highlighting a cell state

The initial step is to determine if the information from an incoming cell state has to be deleted. This is determined by the "forget gate layer". Thereafter, we decide if we have to add any information. This is divided into two steps. An "input Gate layer" will determine the values which are to be updated, afterward a $\tan \mathrm{h}$ section that creates a set of value that is to be added in cell state. Hereafter, they are combined to update the state. To achieve that, we multiply the old state with Function $\mathrm{F}(\mathrm{t})$. And we add to it, It* $C \sim t$. Finally, we employ a sigmoid layer which determines what will be the output. We now use tanh which restricts values to a smaller range. Now we ensure certain selected sections are treated as outgoing output using another gate.

\section{RESULTS AND DISCUSSION}

\subsection{Dataset source}

For the data, we have scrapped the site of SLDC, Delhi. The SLDC is responsible for an integrated power supply to Delhi. The site updates load data every five minutes. To scrap the data, we have used Beautiful soup, a python library used for basic scrapping. It is capable of extracting data from hypertext markup language and extensible markup language documents. We took load data for the last month. The load data is taken every 5 minutes. Figure 6 shows the load data obtained for the $23^{\text {rd }}$ of February 2021 . It can be seen, the load demanded is lesser in the night and peaks during a time span of 9 to 12 o'clock span. In Figure 7 we see the variation of load from $28^{\text {th }}$ of January 2021 till $28^{\text {th }}$ of February 2021. It shows the entire 30-day period load. 


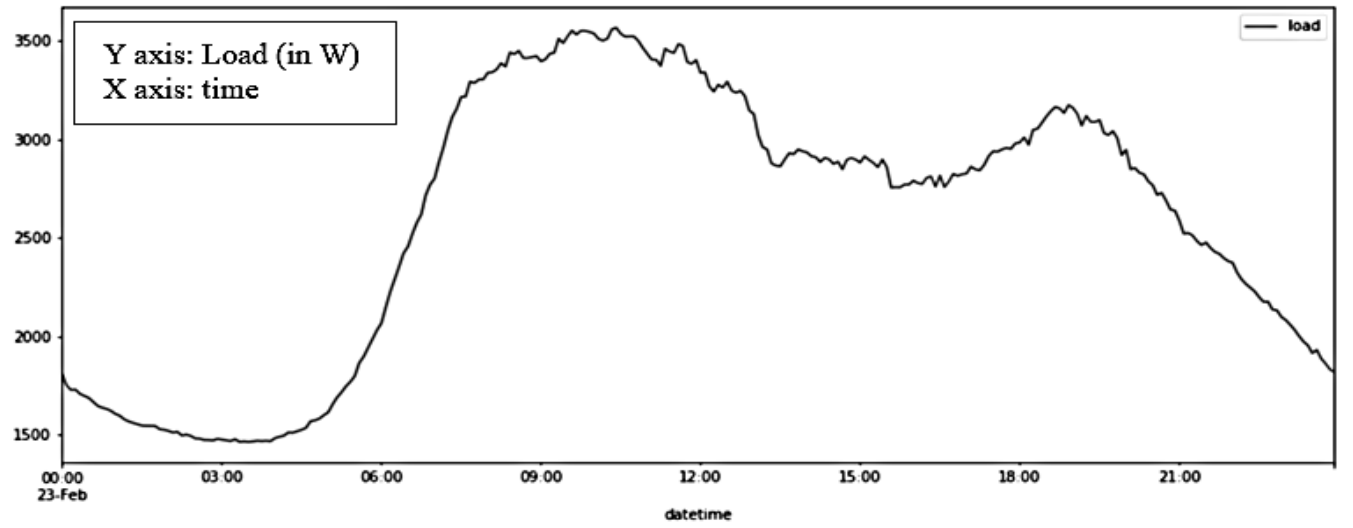

Figure 6. Load data on a particular date

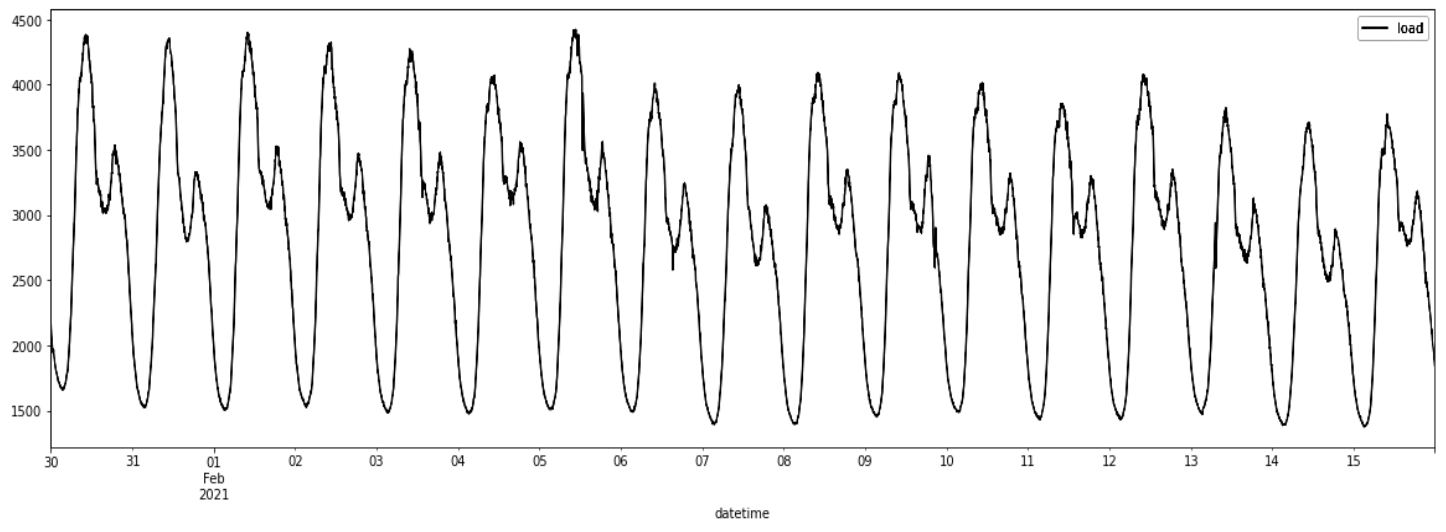

Figure 7. Variation of load in a particular month

\subsection{Data cleaning and preparation}

Now using seasonal decompose from Python's Stats model library, we decompose the data (using daily frequency as a basis) into trends, seasonality, and residue. Figure 8 shows the results of using seasonal decompose, the topmost is the actual observed data, the next section shows the prevalent trend and then the regularity of structure is shown by the seasonal part. The next section is the residual part.
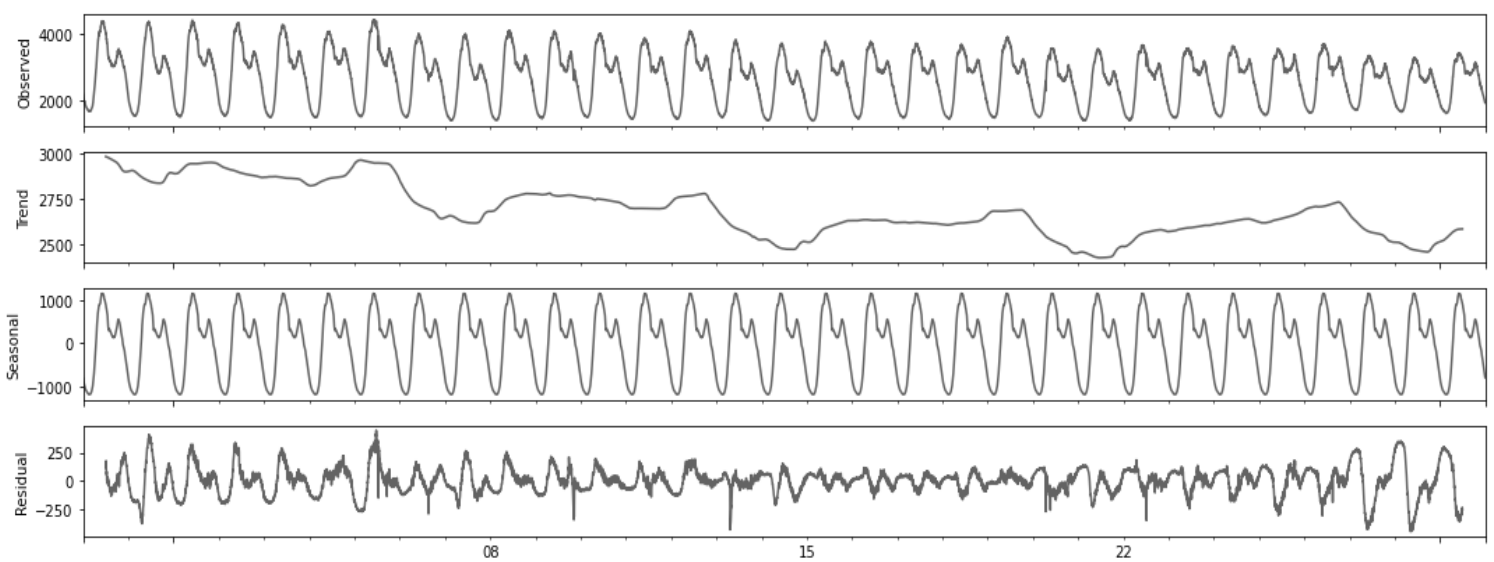

Feb
2021

datetime

Figure 8. Observed, trend, seasonal, and residual data 


\subsection{Identifying the trend of the time series data set and detrending}

Figure 8 shows the trend of data, which can be considered to be a time series data set. A trend is defined as a regular increase and decrease of values over the mean. The trend present in the data set is of stochastic type. Panda et al. [25] argued that detrending can reduce errors in forecasts and improve overall performance. Thus removal of this trend can improve the forecasting ability of the model. The removal of a trend is called detrending. Detrending must be done with proper methods else becomes detrimental. Detrending doesn't always improve performance, specifically for machine learning applications. However, we have chosen to detrend our data because it has proven to be beneficial for time series forecasting [26].

\subsection{Removing seasonality and rescaling the data}

Seasonality refers to regularly repeating patterns in the data set. Seasonal components tend to obscure the actual data pattern that is significant for modeling [27], [28]. In this work, a different method to remove seasonality for our data set. Now before the data set can be used for training and fitting into the network, it should be scaled down to much lower values so that those processing are faster and more efficient [29], [30]. We have scaled down our data to lie between -1 to 1 . Figure 9 shows the data after detrending, removal of seasonality, and rescaling.

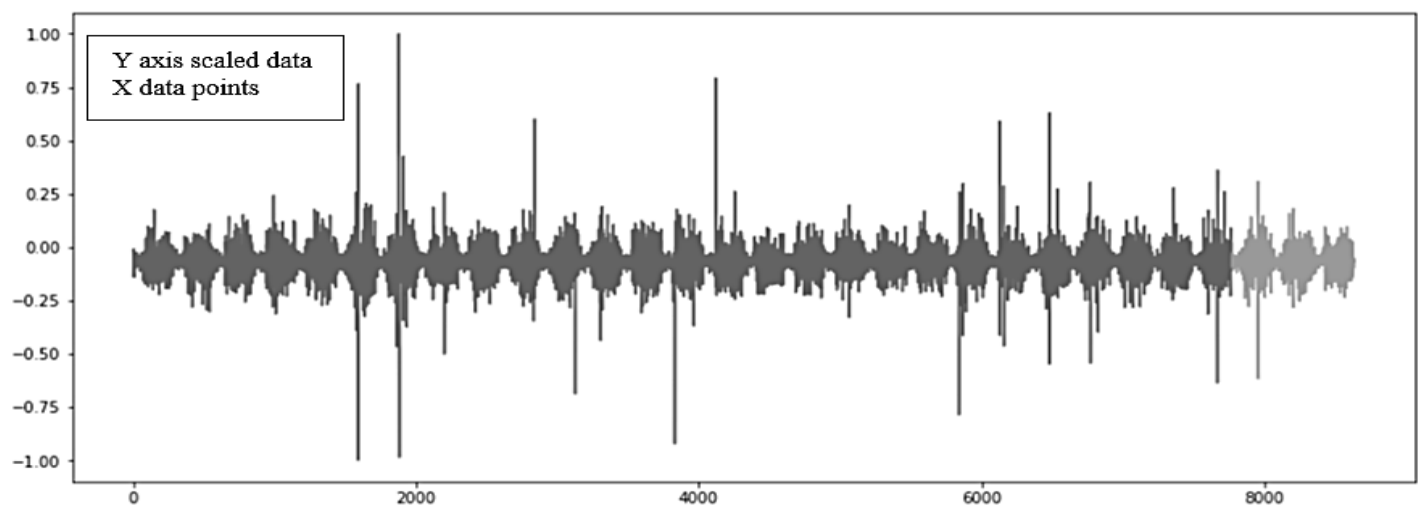

Figure 9. Detrended and rescaled data

\subsection{Model training and forecast}

First data is reshaped and treated to start the model training. From Keras layers, we directly invoke LSTM and from Keras. Models we invoke sequentially, now we have to decide the epochs and batch size [31], [32]. We have decided to run the model training for 30 epochs the batch size has been taken as one. After training for 30 epochs and cross-validating as well, the model is ready for making forecasts. Figure 10 shows the load forecast. Root mean square error (RMSE) or root mean squared error is one of the standard error parameters when only two dimensions are involved. In this work, RMSE is selected as it doesn't get affected by the curse of dimensionality.

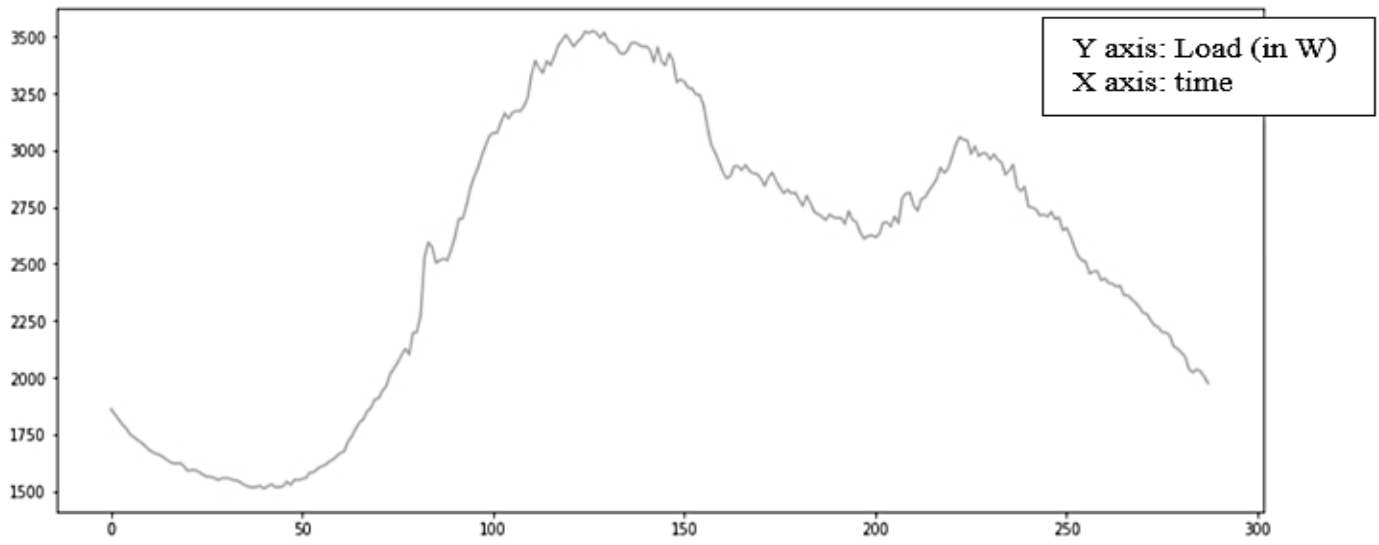

Figure 10. Load forecast 


\subsection{Comparison of forecast with actual load}

Figure 11 shows a comparison between forecasts and actual load. The forecast is depicted in orange color, while the blue graph represents actual load [33]. It shows appreciable accuracy except for a distinct region located left to the middle mark.

The RMSE evaluation reveals an error of $127 \mathrm{~W}$, which is well within the range of appreciable accuracy, is about $4.1 \%$ to $3.2 \%$ of the observed range of peak load experienced in a day. Thus long short term neural network models show significant accuracy in terms of load forecasting. However, further accuracy is required to ensure real life application in actual power plants where a difference of 4.1 to $3.2 \%$ might imply a difference of the magnitudes of 1000 s of KWs.

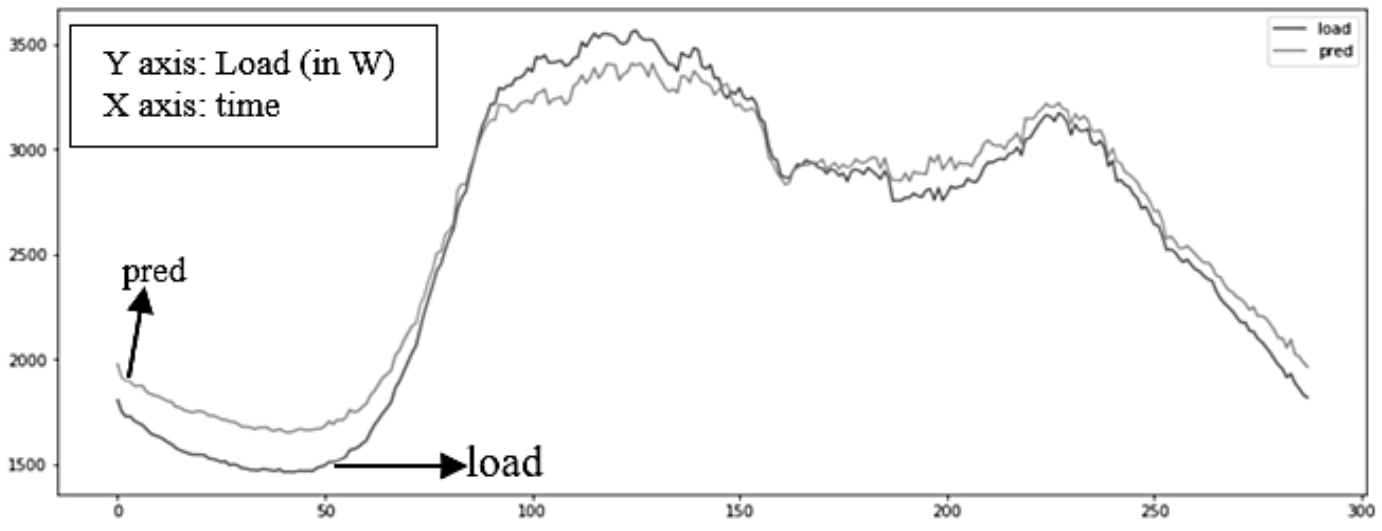

Figure 11. Comparison between actual load and load forecast

\section{CONCLUSION}

LSTM shows appreciable accuracy for electrical load forecasts. It outperforms traditional statistical prediction models and also outperforms many earlier used standard RNN methods. However, room for error persists. Therefore, LSTM can be enhanced with the addition of other techniques. The LSTM model supported by pinball loss results in better performance than a standard one. Another method is to use various optimizers to improve the performance of the LSTM network and then using it for the forecasts. LSTMs can prove to be quite efficient for load forecasts, especially if further improvements are applied to the model. The proposed methodology is subjected to a dynamic data set. From the results it can be concluded that LSTM as a single model is relatively more suitable for electrical load forecasting than traditional methods. Moreover, its superiority might improve beyond other AI techniques with the use of correct optimizers.

\section{ACKNOWLEDGEMENTS}

This research work was funded by “Woosong University’s Academic Research Funding-2021”.

\section{REFERENCES}

[1] J. Mladenovic, V. Lepojevic, and V. J. Milic, "Modelling and prognosis of the export of the Republic of Serbia by using seasonal Holt-Winters and ARIMA method," Economic Themes, vol. 54, no. 2, pp. 233-260, June 2016, doi: 10.1515/ethemes-2016-0012.

[2] Y. Kim, H. Son, and S. Kim, "Short term electricity load forecasting for institutional buildings," Energy Reports, vol. 5, pp. 1270-1280, Nov. 2019, doi: 10.1016/j.egyr.2019.08.086.

[3] M. Tariq and H. V. Poor, "Electricity theft detection and localization in grid-tied microgrids," IEEE Transactions on Smart Grid, vol. 9, no. 3, pp. 1920-1929, Aug. 2016, doi: 10.1109/tsg.2016.2602660.

[4] M. Ali, M. Adnan, and M. Tariq, "Optimum control strategies for short term load forecasting in smart grids," International journal of electrical power \& Energy systems, vol. 113, pp. 792-806, Dec. 2019, doi: 10.1016/j.ijepes.2019.06.010.

[5] V. Gupta and S. Pal, "An overview of different types of load forecasting methods and the factors affecting the load forecasting," International Journal for Research in Applied Science \& Engineering Technology, vol. 5, no. 4, pp. 729-733, Apr. 2017, doi: 10.22214/ijraset.2017.4132.

[6] A. Kavousian, R. Rajagopal, and M. Fischer, "Determinants of residential electricity consumption: Using smart meter data to examine the effect of climate, building characteristics, appliance stock, and occupants' behavior," Energy, vol. 55, pp. 184-194, 2013, doi: 10.1016/j.energy.2013.03.086.

[7] M. Santamouris, N. Papanikolaou, I. Livada, and I. Koronakis, "On the impact of urban climate on the energy consumption of buildings," Solar energy, vol. 70, no. 3, pp. 201-216, Jan 2001, doi: 10.1016/S0038-092X(00)00095-5.

[8] B. Xue and J. Geng, "Dynamic transverse correction method of middle and long term energy forecasting based on statistic of forecasting errors," Proceedings of Conference on Power \& Energy IPEC, Dec 2012, pp. 253-256, doi: 10.1109/asscc.2012.6523273. 
[9] L. Hernández, C. Baladron, J. M. Aguiar, and L. Calavia, “Experimental analysis of the input variables' relevance to forecast next day's aggregated electric demand using neural network," Energies, vol. 6, no. 6, pp. 2927-2948, Jun 2013, doi: $10.3390 /$ en6062927.

[10] S. K. Panda, P. Ray, and D. P. Mishra, "Short Term Load Forecasting using Metaheuristic Techniques," IOP Conf. Series: Materials Science and Engineering, vol. 1033, 2021, pp.1-10, doi: 10.1088/1757-899X/1033/1/012016.

[11] S. Parkpoom, G. P. Harrison, and J. W. Bialek, "Climate change impacts on electricity demand," Universities Power Engineering Conference, vol. 3, Sept. 2014, pp. 1342-1346, doi: 10.1109/tpwrs.2008.922254.

[12] K. B. Sahay and M. M. Tripathi, "Day ahead hourly load forecast of PJM electricity market and iso new england market by using artificial neural network," Proceedings of Innovative Smart Grid Technologies Conference, vol. 3, Sept. 2004, pp. 1342-1346, doi: 10.1109/isgt-asia.2013.6698744.

[13] S. K. Panda, P. Ray, and D. P. Mishra, "Short Term Load Forecasting Using Empirical Mode Decomposition (EMD), Particle Swarm Optimization (PSO) and Adaptive Network-Based Fuzzy Interference Systems (ANFIS)," Innovations in Bio-Inspired Computing and Applications. IBICA 2019. Advances in Intelligent Systems and Computing, vol. 1180. Springer, 2019, pp. 161168, doi: 10.1007/978-3-030-49339-4_17.

[14] Y. Kim, H. Son, and S. Kim, "Short term electricity load forecasting for institutional buildings," Energy Reports, vol. 5, pp. 1270-1280, Nov. 2019, doi: 10.1016/j.egyr.2019.08.086.

[15] B. J. Chen and M. W. Chang, "Load forecasting using support vector machines: A study on EUNITE competition 2001," IEEE transactions on power systems, vol. 19, no. 4, pp. 1821-1830, Nov. 2004, doi: 10.1109/tpwrs.2004.835679.

[16] M. H. Jifri, E. E. Hassan, and N. H. Miswan, "Forecasting performance of time series and regression in modeling electricity load demand," 7th IEEE International Conference on System Engineering and Technology, Oct. 2017, pp. 12-16, doi: 10.1109/icsengt.2017.8123412.

[17] C. Deb, L. S. Eang, J. Y. Yang, and M. Santamouris, "Forecasting diurnal cooling energy load for institutional buildings using Artificial Neural Networks," Energy and Buildings, vol. 121, pp. 284-297, Jun. 2016, doi: 10.1016/j.enbuild.2015.12.050.

[18] H. Shi, M. Xu, and R. Li, "Deep learning for household load forecasting-A novel pooling deep RNN," IEEE Transactions on Smart Grid, vol. 9, no. 5, pp. 5271-5280, Mar. 2017, doi: 10.1109/tsg.2017.2686012.

[19] F. Fahiman, S. M. Erfani, and S. Rajasegarar, "Improving load forecasting based on deep learning and K-shape clustering," International Joint Conference on Neural Networks, May 2017, pp. 4134-4141, doi: 10.1109/ijcnn.2017.7966378.

[20] S. K. Panda, P. Ray, and D. P. Mishra, "A Study of Machine Learning Techniques in Short Term Load Forecasting Using ANN," Intelligent and Cloud Computing. Smart Innovation, Systems and Technologies, vol. 194, 2021, doi: 10.1007/978-981-15-5971-6_6.

[21] K. Zheng, Q. Chen, Y. Wang, and C. Kang, "A novel combined data-driven approach for electricity theft detection," IEEE Transactions on Industrial Informatics, vol. 15, no. 3, pp. 1809-1819, Oct. 2018, doi: 10.1109/tii.2018.2873814.

[22] C. Worrell, L. Luangkesorn, and J. Haight, "Machine learning of fire hazard model simulations for use in probabilistic safety assessments at nuclear power plants," Reliability Engineering \& System Safety, vol. 183, pp. 128-142, Mar 2019, doi: 10.1016/j.ress.2018.11.014

[23] P. Hundi and R. Shahsavari, "Comparative studies among machine learning models for performance estimation and health monitoring of thermal power plants," Applied Energy, vol. 265, May 2020, doi: 10.1016/j.apenergy.2020.114775.

[24] S. K. Panda, P. Ray, and D. P. Mishra, "An Efficient Short-Term Electric Power Load Forecasting Using Hybrid Techniques," International Journal of Computer Information Systems and Industrial Management Applications, vol. 12, pp. 387-397, Nov. 2020, doi: 10.1109/icit48102.2019.00011.

[25] S. K. Panda, P. Ray, and D. P. Mishra, "Effectiveness of GA on Short Term Load Forecasting," International Conference on Information Technology, 2019, pp. 27-32, doi: 10.1109/ICIT48102.2019.00011.

[26] J. Lu, G. J. Carbone, and J. Haight, "Detrending crop yield data for spatial visualization of drought impacts in the United States, 1895-2014," Agricultural and forest meteorology, vol. 237, pp. 196-208, May 2017, doi: 10.1016/j.agrformet.2017.02.001.

[27] S. S. Reddy and J. A. Momoh, "Short term electrical load forecasting using back propagation neural networks," North American Power Symposium (NAPS), pp. 1-6, 2014, doi: 10.1109/naps.2014.6965453.

[28] C. J. Willmott and K. Matsuura, "Advantages of the mean absolute error (MAE) over the root mean square error (RMSE) in assessing average model performance," Climate research, vol. 30, no. 1, pp. 79-82, Dec. 2005, doi: 10.3354/cr030079.

[29] T. Chai and R. R. Draxler, "Root mean square error (RMSE) or mean absolute error (MAE)-Arguments against avoiding RMSE in the literature," Geoscientific model development, vol. 7, no. 3, pp. 1247-1250, Jun. 2014, doi: 10.5194/gmd-7-1247-2014.

[30] S. S. Reddy, "Bat algorithm-based back propagation approach for short-term load forecasting considering weather factors," Electrical Engineering, vol. 100, pp. 1297-1303, 2018, doi: 10.1007/s00202-017-0587-2.

[31] Y. Wang, D. Gan, M. Sun, N. Zhang, Z. Lu, and C. Kang, "Probabilistic individual load forecasting using pinball loss guided LSTM," Applied Energy, vol. 235, pp. 10-20, Feb. 2019, doi: 10.1016/j.apenergy.2018.10.078.

[32] S. S. Reddy and C. M. Jung, "Short-term load forecasting using artificial neural networks and wavelet transform," International Journal of Applied Engineering Research, vol. 11, pp. 9831-9836, 2016, doi: 10.1109/naps.2014.6965453.

[33] T. A. Rashid, P. Fattah, and D. K. Awla, "Using Accuracy Measure for Improving the Training of LSTM with Metaheuristic Algorithms," Procedia Computer Science, vol. 140, pp. 324-333, Jan. 2018, doi: 10.1016/j.procs.2018.10.307.

\section{BIOGRAPHIES OF AUTHORS}

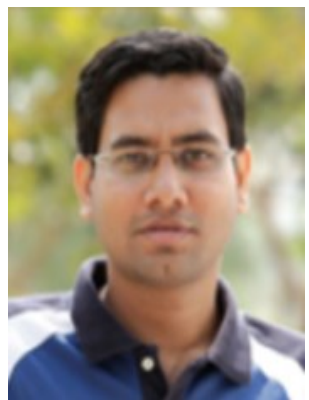

Debani Prasad Mishra (D) SC $\mathrm{P}$ received the B.Tech. in electrical engineering from the Biju Patnaik University of Technology, Odisha, India, in 2006 and the M. Tech in power systems from IIT, Delhi, India in 2010. He has been awarded the Ph.D. degree in power systems from Veer Surendra Sai University of Technology, Odisha, India, in 2019. He is currently serving as Assistant Professor in the Dept. of Electrical Eng., International Institute of Information Technology Bhubaneswar, Odisha. He has 11 years of teaching experience and 2 years of industry experience in the thermal power plant. He is the author of more than 80 research articles. His research interests include soft Computing techniques application in power system, signal processing and power quality. 3 students have been awarded $\mathrm{Ph}$. D under his guidance and currently 4 Ph.D. Scholars are continuing under him. He can be contacted at email: debani@iiit-bh.ac.in. 

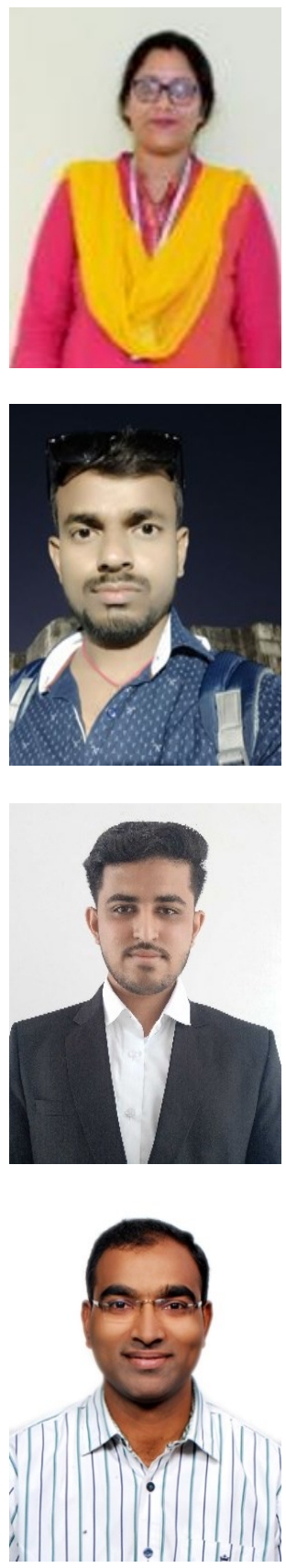

Surender Reddy Salkuti (D) SC P received the Ph.D. degree in Electrical Engineering from the Indian Institute of Technology, New Delhi, India, in 2013. He was a Postdoctoral Researcher with Howard University, Washington, DC, USA, from 2013 to 2014. He is currently an Associate Professor with the Department of Railroad and Electrical Engineering, Woosong University, Daejeon, South Korea. His current research interests include power system restructuring issues, ancillary service pricing, real and reactive power pricing, congestion management, and market clearing, including renewable energy sources, demand response, smart grid development with integration of wind and solar photovoltaic energy sources, artificial intelligence applications in power systems, and power system analysis and optimization. He can be contacted at email: surender@wsu.ac.kr. 